\title{
Prise en charge conservatrice d'un hydropneumothorax chez une enfant de 4 ans atteinte de pneumonie
}

\author{
Carsten Krueger MD, Theo J. Moraes MD, Stacey Bernstein MD
}

Citation : CMAJ 2021 August 30;193:E1350. doi : 10.1503/cmaj.202468-f

Voir la version anglaise de l'article ici : www.cmaj.ca/lookup/doi/10.1503/cmaj.202468

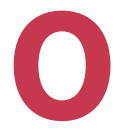

$\mathrm{n}$ a hospitalisé une fillette de 4 ans, en bonne santé et entièrement immunisée, présentant des symptômes de fièvre et de détresse respiratoire perdurant depuis 1 semaine. La radiographie de son thorax montrait une pneumonie du côté droit accompagnée d'une importante effusion pleurale (annexe 1, figure $\mathrm{S} 1$, accessible en anglais au www.cmaj. $\mathrm{ca} /$ lookup/doi/10.1503/cmaj.202468/tab-related-content). On lui a administré de la ceftriaxone et elle a obtenu son congé 7 jours après l'obtention d'un résultat négatif à une hémoculture, accompagnée d'une ordonnance d'un composé d'amoxicilline et d'acide clavulanique administré par voie orale pour une période de 2 semaines. Huit jours après avoir obtenu son congé, une toux et une douleur thoracique au côté droit se sont manifestées et on l'a conduite à l'hôpital à nouveau. Elle semblait bien se porter avec une fréquence cardiaque de 120 battements/min, une fréquence respiratoire de 20 respirations/min, une température de $36^{\circ} \mathrm{C}$ et une saturation en oxygène de $97 \%$. Nous avons observé une diminution des bruits de respiration à l'auscultation du côté droit de son thorax. Une radiographie thoracique montrait un hydropneumothorax au côté droit (figure 1). Nous l'avons hospitalisée afin de l'observer et de procéder à l'administration de ceftriaxone sous forme intraveineuse. Au cours des 2 journées suivantes, les radiographies thoraciques quotidiennes ont montré une amélioration et son état clinique se maintenait. Après une consultation en pneumologie et en radiologie d'intervention, nous avons décidé qu'une intervention ou l'emploi de modalités d'imagerie avancée ne pourraient offrir d'avantages thérapeutiques. La patiente a obtenu son congé de l'hôpital, recevant ultimement des antibiotiques sur une période de 6 semaines tout en demeurant asymptomatique. Elle a aussi subi des radiographies thoraciques de contrôle et elle s'est présentée à des rendez-vous cliniques 5 jours et 2 semaines après l'obtention de son congé. Huit mois plus tard, une radiographie de son thorax était tout à fait normale (annexe 1, figure S2).

L'hydropneumothorax est une complication peu fréquente de la pneumonie caractérisée par l'accumulation de fluide et d'air dans la cavité pleurale dont on croit qu'elle résulte d'une fuite

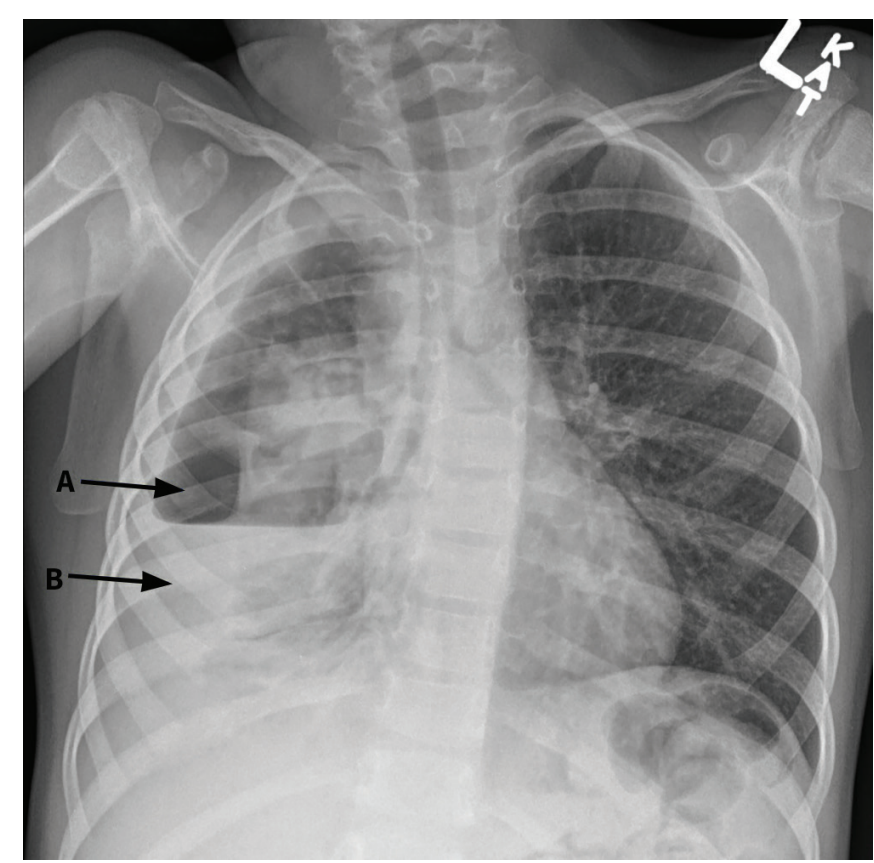

Figure 1 : Radiographie thoracique d'une fillette de 4 ans, prise au cours d'une deuxième hospitalisation et montrant (A) un important collapsus pulmonaire du côté droit et (B) un hydropneumothorax.

d'air à travers le parenchyme pulmonaire nécrotique ${ }^{1}$. Les rares cas pédiatriques mentionnés dans la documentation ont été pris en charge par l'insertion d'un drain thoracique jusqu'à la disparition des symptômes, nécessitant une hospitalisation prolongé $^{1,2}$. Nous avons considéré qu'en raison de l'état clinique satisfaisant de la patiente, un suivi rapproché présentait un faible risque comparativement à l'insertion d'un drain thoracique. Les risques d'une telle intervention chez les enfants comprennent ceux associés à la sédation et à l'anesthésie, à la douleur, au développement de fistules bronchopleurales et à une hospitalisation prolongée ${ }^{3}$. Les drains thoraciques sont clairement indiqués chez les patients présentant un hydropneumothorax compressif, une détérioration de l'hydropneumothorax ou une détresse respiratoire. Ce cas démontre qu'une prise en charge 
conservatrice, composée d'une observation du patient hospitalisé suivie de radiographies de contrôle et de rendez-vous rapprochés en consultations externes, peut mener à une résolution positive d'une pneumonie aggravée par un hydropneumothorax chez des enfants par ailleurs en bonne santé. Les candidats à une prise en charge conservatrice sont des patients dont l'état est cliniquement stable tout en démontrant des signes d'amélioration au cours d'une série de radiographies thoraciques et dont les tuteurs sont responsables et peuvent soutenir un suivi rapproché en consultation externe.

\section{Références}

1. Miller JC, Boyce TG. Hydropneumothorax as a complication of necrotizing pneumonia in a young girl. Clin Case Rep 2019;7:1559-61.

2. Ortega C, Gonzales C, Soto-Martinez ME, et al. Hydropneumothorax in children: a rare complication of a bacterial pneumonia. Case Rep Pediatr 2016;2016. doi: 10.1155/2016/8097105.

3. Kiliç N, Çelebi S, Gürpınar A, et al. Management of thoracic empyema in children. Pediatr Surg Int 2002;18:21-3.

Intérêts concurrents : Aucun déclaré.

Cet article a été révisé par des pairs.

Les auteurs ont obtenu le consentement de la famille.

Affiliations : Département de pédiatrie, Faculté de médecine Temerty, Université de Toronto, Toronto, Ont.

Propriété intellectuelle du contenu : Il s'agit d'un article en libre accès distribué conformément aux modalités de la licence Creative Commons Attribution (CC BY-NC-ND 4.0), qui permet l'utilisation, la diffusion et la reproduction de tout médium à la condition que la publication originale soit adéquatement citée, que l'utilisation se fasse à des fins non commerciales (c.-à-d., recherche ou éducation) et qu'aucune modification ni adaptation n'y soit apportée. Voir : https://creativecommons.org/licenses/by-nc-nd/4.0/deed.fr.

Correspondance : Stacey Bernstein, Stacey.bernstein@sickkids.ca

Les images cliniques sont choisies pour leur caractère particulièrement intéressant, classique ou impressionnant. Toute soumission d'image de haute résolution claire et bien identifiée doit être accompagnée d'une légende aux fins de publication. On demande aussi une brève explication ( 300 mots maximum) de la portée éducative des images, et des références minimales. Le consentement écrit du patient au regard de la publication doit être obtenu avant la soumission. 